\title{
A Novel RP-HPLC Method Development and Validation for Estimation of Nicoumalone in Bulk drug and Formulation
}

\author{
Thota Anusha*1, Thota Prasad', Valluri Asha Ranjani², Sahu Puranlal ${ }^{1}$, Kumar Robin', Singh \\ Gyanendra Nath ${ }^{1}$
}

${ }^{1}$ Reference Standard Division, Indian Pharmacopoeia Commission, Ghaziabad-201002, INDIA.

${ }^{2}$ Department of Pharmaceutical Analysis, MLR Institute of pharmacy, Dundigal-500043, INDIA.

\begin{abstract}
Objective: The objective of this study is to validate a simple, precise, and accurate isocratic reverse phase high performance liquid chromatography (RP-HPLC) method for estimation of nicoumalone in bulk and tablet dosage form. Method \& Results: The quantification is carried out by using C18 column (inertsilR ODS-3V column, $250 \times 4.6$ $\mathrm{mm}, 5 \mu \mathrm{m}$ ). A mixture of Acetonitrile: Potassium dihydrogen phosphate buffer $10 \mathrm{mM}, \mathrm{pH}$ 6 adjusted using $0.1 \mathrm{~N} \mathrm{KOH}$ in $50: 50 \mathrm{v} / \mathrm{v}$ ratio used as mobile phase. The flow rate was set at $1 \mathrm{ml} / \mathrm{min}$ and the Separation performed at ambient temperature. UV detector set at $283 \mathrm{~nm}$. The retention time of nicoumalone found to be 4-5 minutes. Method Validation: Validation of proposed method is done as per International Conference of Harmonization guidelines (ICH). The analytical parameters are statistically validated for linearity and range, precision, limit of detection, limit of Quantitation, accuracy, and robustness. Conclusion: The proposed method is useful for best analysis of pharmaceutical dosage forms.
\end{abstract}

Key words: Nicoumalone, RP-HPLC method and Validation, ICH, Estimation, Dosage Form.

\section{INTRODUCTION}

Nicoumalone is a coumarin derivative used as an anticoagulant. Coumarin derivatives inhibit the reduction of Vitamin K by Vitamin K reductase. Nicoumalone is an oral anticoagulant that functions as a Vitamin $\mathrm{K}$ antagonist. Chemically it is 4-hydroxy-3-(1-(4- nitro phenyl)-3-oxobutyl)-Coumarin (Figure 1). It is administered in the management of thromboembolic disorders. More specifically, it is indicated for the prevention of cerebral embolism, pulmonary embolism, thromboembolism in infarction and transient ischemic attacks. It is used for the treatment of deep vein thrombosis and myocardial infarction. Nicoumalone (also known as Acenocoumarol) is official in Indian pharmacopoeia. ${ }^{1}$ and British pharmacopoeia. ${ }^{2}$ Both the pharma- copoeias explain the assay of Acenocoumarol API by aqueous acid base titration while that of the tablet formulation by Spectrophotometric method $\left(\lambda_{\max }\right.$ at $306 \mathrm{~nm}$ and specific absorbance at 521). ${ }^{3}$ Extensive literature survey revealed that a few spectroscopic, 4,5 HPLC, ${ }^{6,7}$ and bio-analytical, ${ }^{8-12}$ methods have been reported for its quantitative estimation in pharmaceutical formulations and biological fluids, which include Spectrophotometric, and bioanalytical methods. Current research work mainly devoted to develop a simple, rapid, precise, accurate and reproducible isocratic RP-HPLC method for the determination of nicoumalone and the developed method is validated with respect to specificity, linearity, precision, accuracy, range,
Submission Date : 20-01-2016 Revision Date : :04-04-2016 Accepted Date : :02-05-2016

DOI: 10.5530/ijper.50.3.13 Correspondence:

Thota Anusha,

Reference StandardDivision, Indian Pharmacopoeia

Commission,

Ghaziabad-201002, INDIA

Phoneno:0120-2378400

E-mail:prasad.thota187@

gmail.com

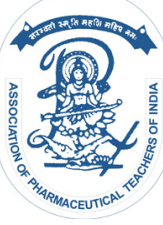

www.ijper.org 
LOD, LOQ and robustness. The method validated in accordance with International Conference on Harmonization $(\mathrm{ICH})$ guidelines.

\section{MATERIALS \& METHODS}

\section{HPLC Instrumentation and conditions}

The RP-HPLC method development and partial validation studies are performed using Agilent 1200 system, comprising of a quaternary solvent delivery module, online degasser, column thermostat, auto sampler, UV detector. The separation achieved by isocratic elution. The mobile phase comprising of Acetonitrile: potassium dihydrogen phosphate buffer $10 \mathrm{mM}, \mathrm{pH} 6$ (adjusted using $0.1 \mathrm{~N} \mathrm{KOH}$ ) 50:50 was delivered at a flow rate of $1 \mathrm{ml} / \mathrm{min}$ and the column eluent was monitored at $283 \mathrm{~nm}$ with the injection volume of $10 \mu$ l. The mobile phase and samples were filtered using $0.2 \mu \mathrm{m}$ membrane filter and degassed using an ultrasonicator (MODEL: BRANSON 1510) before injecting in HPLC system. Inertsil $^{\mathrm{R}}$ ODS-3V column $(4.6 \times 250 \mathrm{~mm}, 5 \mu \mathrm{m})$ utilized for analysis; Chromatogram output, integration of peaks and calculation of peak areas, retention times, system suitability parameters such as peak asymmetry column efficiency etc were obtained using the EZ chrome elite software.

\section{Chemicals and Reagents}

Pharmaceutical grade nicoumalone obtained from Indian Pharmacopoeia Commission Ministry of health \& Family welfare, Government of India, Indian Pharmacopoeia Laboratory. It is contain $98.98 \% \mathrm{w} / \mathrm{w}$ of Nicoumalone on dried basis and used as such without further purification. All chemicals and reagents used were of HPLC grade, High-purity water was prepared using Millipore purification system. Commercial formulation of nicoumalone (ACITROM, Abbott Healthcare Pvt. Ltd) purchased from local medicine store.

\section{Preparation of stock and standard solutions}

A stock solution of nicoumalone and internal standard were prepared by accurately weighing $50 \mathrm{mg}$ of drug, transferring to $50 \mathrm{ml}$ of volumetric flask, containing $50 \mathrm{ml}$ of methanol dissolving it to obtain final stock solution of $1000 \mu \mathrm{g} / \mathrm{ml}$ concentration of nicoumalone or internal standard. Resultant solution filtered through sample filter paper. This solution is further diluted with the same solvent to obtain concentrations in the range of $20-120 \mu \mathrm{g} / \mathrm{ml}$. These solutions were used for analysis.

\section{Preparation of sample solution for assay}

20 tablets containing label claim of $1 \mathrm{mg}$ weighed accurately and average weight of tablet was determined.
A quantity of the powder equivalent to $71.936 \mathrm{mg}$ of nicoumalone dissolved in $100 \mathrm{ml}$ of methanol with the help of ultrasonication to prepare a solution of concentration $10 \mu \mathrm{g} / \mathrm{ml}$.

\section{Method Development}

The HPLC procedure optimized with a view to developing a method for Assay. No internal standard used because no extraction or separation step was involved. Of several solvents and solvent mixtures investigated Acetonitrile-Phosphate buffer $\left(\mathrm{P}^{\mathrm{H}}-6\right)$ 50:50 (\%,v/v) was found to furnish sharp, well-defined peaks with very good symmetry (1.36) and low $\mathrm{t}_{\mathrm{R}}(5.86 \mathrm{~min}$ ) (Figure 2). With Acetonitrile-water 50:50 as mobile phase $t_{R}$ was 1.5 min (Figure 3) and peak shape and sensitivity were poor. Acetonitrile-water 40:60 (Figure 4) fronting and tailing effects are observed with very low number of theoretical plates and Acetonitrile-Phosphate buffer 50:50 (Figure 5), Acetonitrile-Phosphate buffer 45:55 (Figure 6), did not furnish sharp, well-defined peaks and other mobile phases tried either resulted in much lower sensitivity or did not give well defined peaks in a short time, and hence were not considered. The final decision on mobile phase composition and flow rate was made on the basis of peak shape (peak area, asymmetry, tailing factor), baseline drift, time required for analysis, and cost of solvent. Acetonitrile-Phosphate buffer 50:50 $\mathrm{P}^{\mathrm{H}}-6$ $(\%, v / v)$ selected as the optimum mobile phase. Under these conditions the retention time and asymmetry factor were $5.86 \pm 0.01 \mathrm{~min}$ and $1.36 \pm 0.03$, respectively.

\section{Validation of the developed method}

The developed method was validated, ${ }^{13,14}$ as per ICH guidelines for the following parameters (Table 2).

\section{Linearity and Range}

The stock solution $1000 \mu \mathrm{g} / \mathrm{ml}$ of nicoumalone was prepared in methanol and this stock solution diluted with methanol to obtain final concentrations $20-120 \mu \mathrm{g} / \mathrm{ml}$. $10 \mu \mathrm{l}$ volume of each concentration injected into LC for six times, under the optimized conditions. The prepared dilutions injected in series, peak area calculated for each dilution, and concentration plotted against peak area.

\section{Precision}

The precision for the quantification of nicoumalone by RP- HPLC is verified by repeatability (system precision $\&$ method precision).

\section{System precision}

The system precision was performed by injecting six replicate injections of the standard solution in to the Chromatography (Figure 8). The \% RSD for the peak 


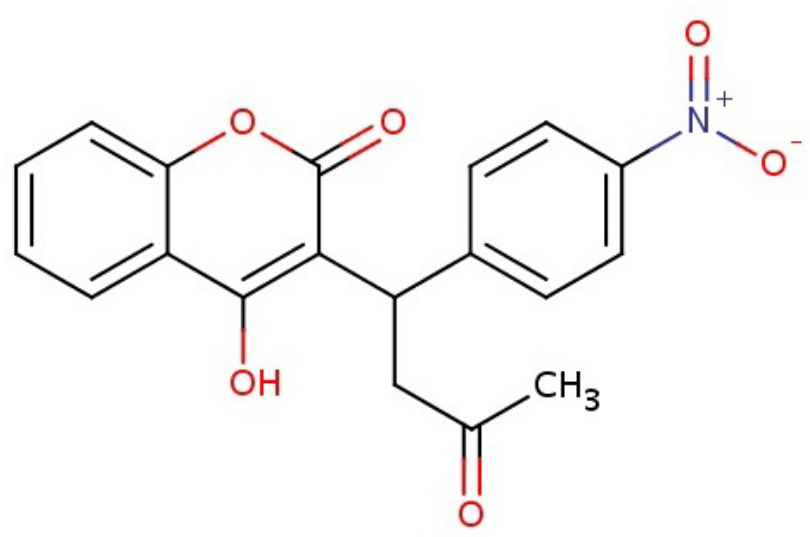

Figure 1: Structure of Nicoumalone.

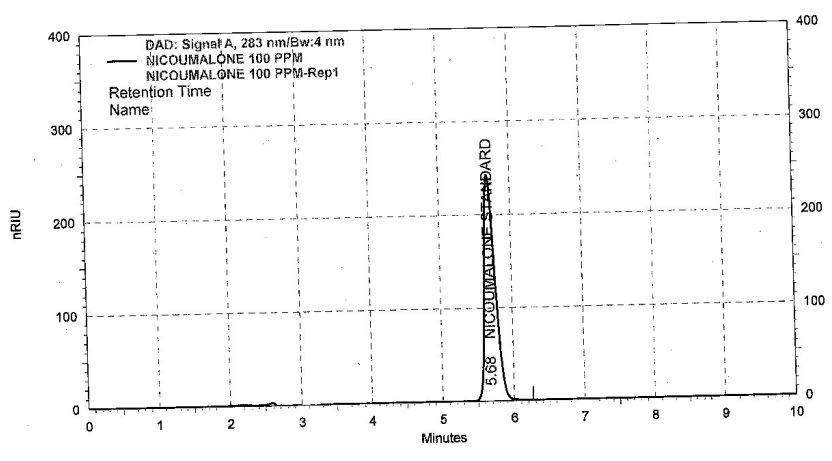

Figure 2: HPLC chromatogram obtained from nicoumalone with Acetonitrile - phosphate buffer $\left(\mathrm{P}^{\mathrm{H}}-6\right)$ 50:50 (\% v/v), $t_{\mathrm{R}} 5.68 \mathrm{~min}$.

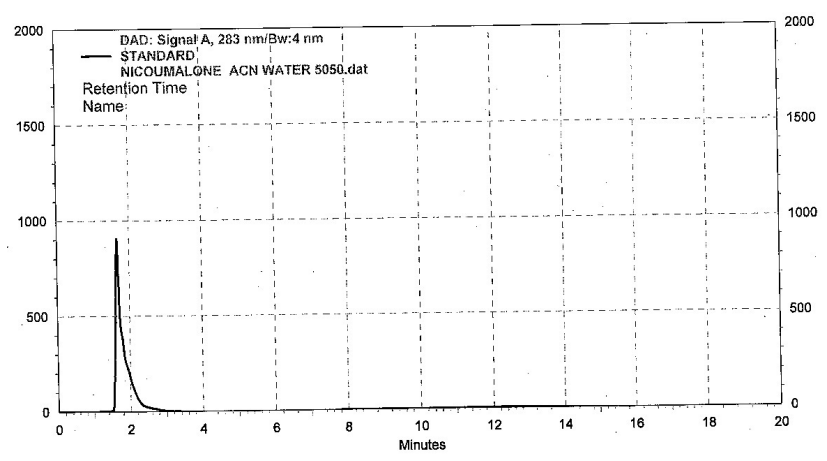

Figure 3: HPLC chromatogram obtained from nicoumalone with Acetonitrile-water $50: 50$ as mobile phase $t_{R}$ was 1.5 min.

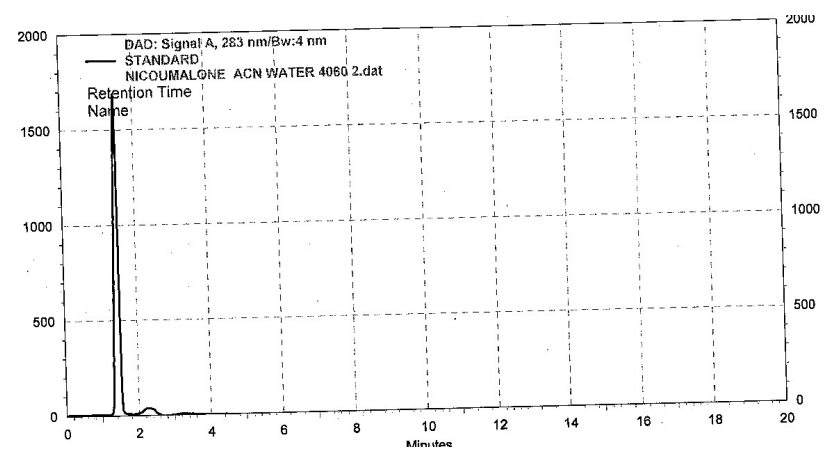

Figure 4: HPLC chromatogram obtained from nicoumalone with Acetonitrile-water 40:60.

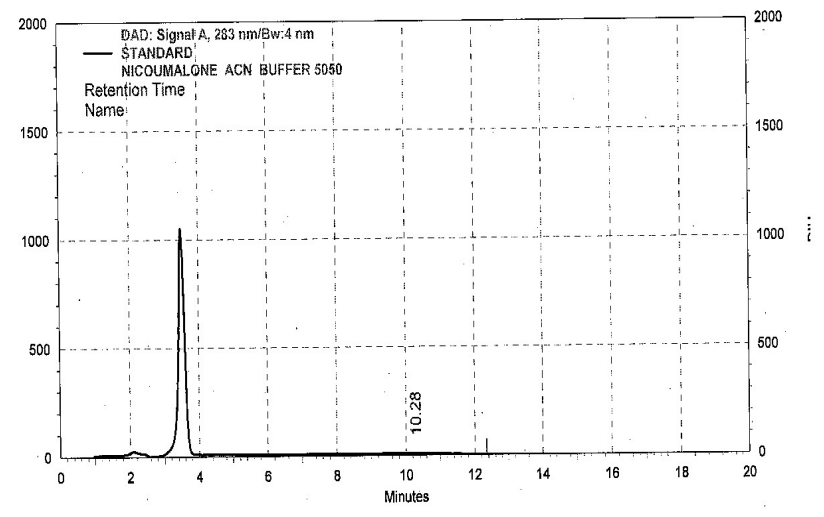

Figure 5: HPLC chromatogram obtained from nicoumalone with Acetonitrile-Phosphate buffer 50:50.

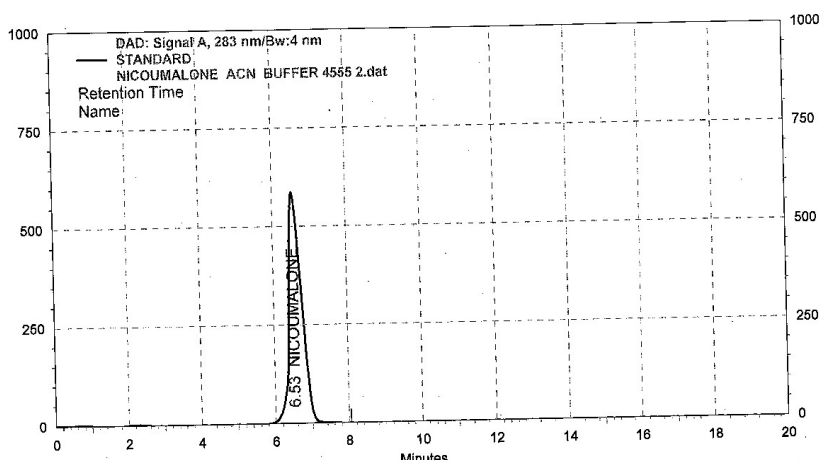

Figure 6: HPLC chromatogram obtained from nicoumalone with Acetonitrile-Phosphate buffer 45:55.

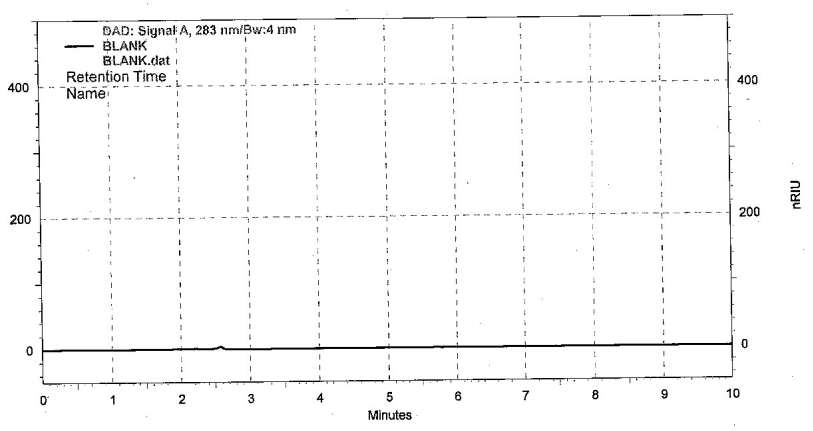

Figure 7: RP-HPLC chromatogram of blank solution at $283 \mathrm{~nm}$.

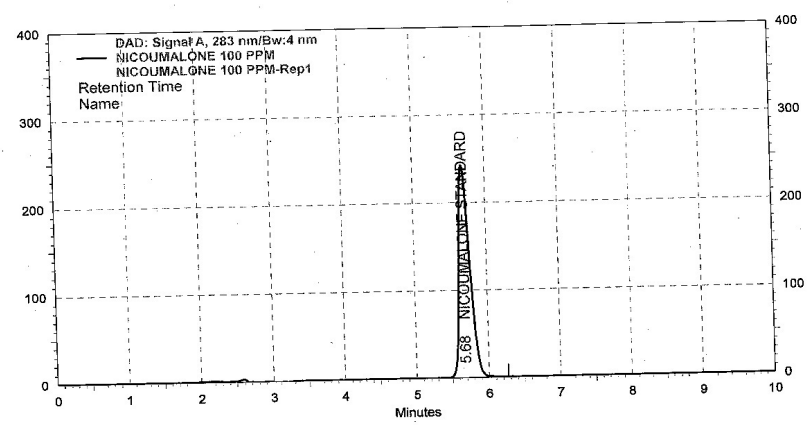

Figure 8: RP-HPLC chromatogram of nicoumalone standard solution at $283 \mathrm{~nm}$. 


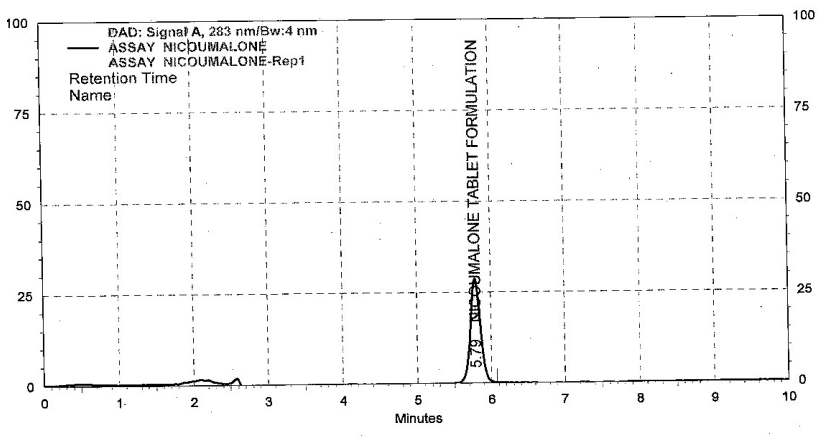

Figure 9: RP-HPLC chromatogram of nicoumalone tablet solution at $283 \mathrm{~nm}$.

\begin{tabular}{|c|c|c|}
\hline \multicolumn{3}{|c|}{ Table 1: linearity of Nicoumalone } \\
\hline S.No. & Conc. $(\boldsymbol{\mu g} / \mathrm{ml})$ & Area \\
\hline 1 & 20 & 1127340.33 \\
\hline 2 & 40 & 2206973.33 \\
\hline 3 & 60 & 3244648 \\
\hline 4 & 80 & 4436337.16 \\
\hline 5 & 100 & 5326015.83 \\
\hline 6 & 120 & 6347491.83 \\
\hline
\end{tabular}

\begin{tabular}{|c|c|}
\hline \multicolumn{2}{|c|}{ Table 2: Summary of Validation Parameters } \\
\hline Parameter (Units) & Results \\
\hline Linearity range $(\mu \mathrm{g} / \mathrm{ml})$ & $20-120$ \\
\hline Correlation Coefficient & 0.998 \\
\hline Slope & 52356.53 \\
\hline Intercept & 116510.33 \\
\hline Recovery $(\%)$ & 100 \\
\hline Precision (\%RSD) & \\
\hline System Precision & 0.004 \\
\hline Method Precision & 0.004 \\
\hline Robustness & Robust \\
\hline Retention Time & 5.68 \\
\hline Tailing Factor (Asymmetry Factor) & 1.09 \\
\hline Theoretical Plates & 8444 \\
\hline
\end{tabular}

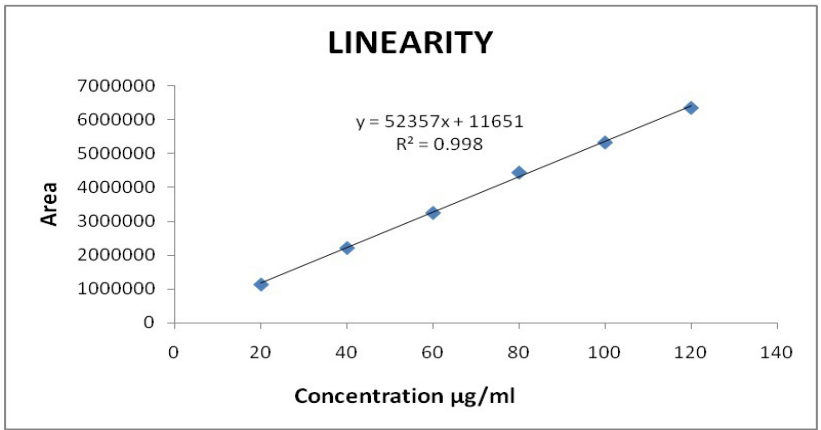

Figure 10: Calibration graph of Nicoumalone.

of interest was calculated and found less than $1 \%$ (should be less than 2).

\section{Method precision}

The method precision performed by preparing six replicate sample preparations as per testing procedure and injected in to the chromatography (Figure 9). The percentage $\mathrm{w} / \mathrm{w}$ of nicoumalone calculated from six replicates and $\%$ RSD.

\section{Accuracy}

The accuracy of an analytical method is the closeness of results obtained by that method to the true value for the sample. It is expressed as recovery $(\%)$, and determined by the standard addition method. Samples were spiked with 80,100 and $120 \%$ of the standard and Analyzed. The experiment performed in triplicate. Recovery (\%) and RSD (\%) were calculated for each concentration.

\section{Limit of Detection (LOD) and Limit of Quantitation (LOQ)}

LOD and LOQ of nicoumalone were separately determined based on the standard deviation of response of the respective calibration curves.

\begin{tabular}{|c|c|c|c|c|c|}
\hline Level & $\begin{array}{c}\text { Theoretical } \\
\text { Amount }(\mu \mathrm{g} / \mathrm{ml})\end{array}$ & Peak area & \% Recovery & $\begin{array}{l}\text { Average } \\
\text { Recovery }\end{array}$ & $\%$ RSD \\
\hline 80 & 180 & 50795752 & 95 & \multirow{3}{*}{100} & \multirow{3}{*}{0.09539} \\
\hline 80 & 180 & 50746006 & 94 & & \\
\hline 80 & 180 & 50850241 & 111 & & \\
\hline 100 & 200 & 54073587 & 105 & \multirow{3}{*}{104.333} & \multirow{3}{*}{0.00553} \\
\hline 100 & 200 & 54240135 & 104 & & \\
\hline 100 & 200 & 54349591 & 104 & & \\
\hline 120 & 220 & 64877937 & 99 & \multirow{3}{*}{97.3333} & \multirow{3}{*}{0.01569} \\
\hline 120 & 220 & 66096450 & 96 & & \\
\hline 120 & 220 & 65419497 & 97 & & \\
\hline
\end{tabular}




\section{Robustness}

The robustness of an analytical method is a measure of its capacity to remain unaffected by small but deliberate variations in method parameters and provides an indication of its reliability during normal usage. It is concluded that the method is robust as it is found that the $\%$ RSD is less than 1 for both the drugs concerning $\%$ assay despite deliberate variations done concerning flow rate $( \pm 0.2), \mathrm{pH}( \pm 0.2)$ and $\%$ organic phase composition $( \pm 2 \%)$.

\section{Assay of Acenocoumarol in tablet formulation}

Assay was performed by using the regression equation $\left(y=52357 x+116510, r^{2}=0.9988\right)$ obtained from the standard calibration curve of nicoumalone API. The percentage purity found to be $100.04 \%$ as per the method.

\section{RESULTS AND DISCUSSIONS}

The current RP-HPLC technique is most accurate, reliable and precise method of analysis for the quantitative estimation of nicoumalone in bulk drugs and its pharmaceutical formulations and dosage form. The current method system suitability was accounted by measuring USP tailing factor, USP Plate count for the peak of nicoumalone from Standard Solution and percentage RSD of Area for the peak of nicoumalone from six replicate injection of Standard Solution. The obtained values are 1.09 as USP tailing factor (should be not more than 2.0), 8444 as USP Plate count (should be not less than 2000), and percentage RSD of area as 0.04 (should be not more than 2.0). The typical nicoumalone retention time in the current developed method was about 6 minutes. All variations resulted with the very good percentage RSD of Assay i.e. less than 1.0\%.

\section{Linearity and range}

The calibration curve showed good linearity in the range of $20-120 \mu \mathrm{g} / \mathrm{ml}$ for nicoumalone API with correlation coefficient $\left(\mathrm{r}^{2}\right)$ of 0.998 (Figure 10). A typical calibration curve has the regression equation $y=52357 x+116510$ for nicoumalone as shown in Table 1.

\section{Precision}

Six replicate injections of the mixture of standard solution at working concentration showed \% RSD (Relative Standard Deviation) less than 2 concerning peak areas of the drug, which indicates the acceptable reproducibility and thereby the precision of the system. System precision results are tabulated in Table 3.

\section{Method precision}

Method precision was determined by performing assay of sample under the test concentration showed $\%$ RSD less than 2 concerning $\%$ assay of the drug which indicate that the method developed is method precise by the test of repeatability and hence can be understood that the method gives consistently reproducible results (Table 3).

System precision: Six consecutive injections of the sample at working concentration showed \% RSD less than 2 concerning $\%$ assay of the drug which indicate that the method developed is system precise by the standard of repeatability and hence can be understood that the method gives consistently reproducible results (Table 3).

\section{Limit of Detection (LOD) and Limit of Quantitation (LOQ)}

LOD and LOQ determined by the standard deviation $\left(\mathrm{S}_{\mathrm{y} / \mathrm{x}}\right)$ method. Blank samples injected in triplicate and the peak area of this blank was calculated (Figure 7). LOD and LOQ were determined from the slope $\mathrm{S}$, of the calibration plot and the standard deviation of the response for the blank sample, $\mathrm{S}_{\mathrm{y} / \mathrm{x}}$, by use of the formulae LOD $=3.3 \times$ $\mathrm{S}_{\mathrm{y} / \mathrm{x}} / \mathrm{S}$ and $\mathrm{LOQ}=10 \times \mathrm{S}_{\mathrm{y} / \mathrm{x}} / \mathrm{S}$. The minimum concentration level at which the analyte can be reliably detected (LOD) and quantified (LOQ) were found to be 1.53 and $4.65 \mu \mathrm{g} / \mathrm{ml}$ respectively as shown in Table 3.

\section{Accuracy}

The mean recovery of the method, determined by standard addition method, and found to be $100 \%$. The values of $\%$ recovery and $\%$ RSD, listed in Table 3 indicate that the method is accurate.

\section{Robustness}

Small changes in chromatographic conditions such as change in flow rate $( \pm 0.2 \mathrm{ml} / \mathrm{min})$, percentage of Acetonitrile in mobile phase $( \pm 2 \%)$ and column temperature $\left( \pm 2{ }^{\circ} \mathrm{C}\right)$, studied to determine the robustness of the method, were also in favour of the developed RP-HPLC method for the analysis of nicoumalone API.

\section{CONCLUSION}

There is no specific and short run time analytical method in literature for nicoumalone moreover; the method gave adequate resolution for the drug with a short analysis time. The developed method was validated as per ICH guidelines. All the validation parameters were found to be well within the acceptance criteria. We conclude that the method was accurate, reproducible, repeatable, linear, precise, and reliable. These results show the method 
could find practical application as a quality control tool for analysis of the drug in its Tablet dosage forms in quality control laboratories.

\section{ACKNOWLEDGEMENTS}

The authors are thankful to Indian Pharmacopoeia Commission, Ministry of Health \& Family welfare, Government of India, for providing the sample of nicoumalone and also for carryout research work in Indian Pharmacopoeia Laboratory.

\section{CONFLICT OF INTEREST}

None.

\section{ABBREVIATION USED}

RP-HPLC: Reverse phase-High performance liquid chromatography; Uv: ultra voilet; ICH: International conference of harmonization; $\mathbf{K O H}$ : Potassium hydroxide; API: Active Pharmaceutical Ingredient; LOD: limit of detection; LOQ: Limit of quantification; RSD: Relative Standard deviation; USP: United state pharmacopoeia; BP: British Pharmacopoeia.

\section{REFERENCES}

1. Indian Pharmacopoeia, Controller of Publication, Government of India, Ministry of Health \& Family Welfare, New Delhi,2014;3:2335-36.

2. British Pharmacopoeia, HMSO publication: London;2014;1:52-53.

3. Supriya N, Mandrupkar, Sugandha V, Mulgund and Madhuri A. Nagras:Development of validated stability indicating rp-hplc method for estimation of Acenocoumarol in bulk and tablet dosage form, Int J Pharm Sci Rev Res. 2012;16(1):101-6.
4. Mehta AS, Gurupadayya BM, Gopinath B, Manohara YN and Chandra A, Spectrophotometric Estimation of Acenocoumarol in Tablets, Indian. J Pharm Educ Res. 2008;42(4):310-13.

5. Mehta AS, Gurupadayya BM, Gopinath B, manohara YN and Chandra A. Spectrophotometric methods for estimation of acenocoumarol in bulk and its pharmaceutical dosage forms. Int J Chem Sci. 2008;6(2):1067-73.

6. Ceresole R, Rosasco MA, Forastieri CC and Segall AI. HPLC Determination of Acenocoumarol and its Major Thermal Degradation Product. J Liq Chromaogra \& Rel Tech. 2008;31(2):179-87.

7. Orsi DD, Gagliardi L, Turchetto $L$ and Tonelli D. HPLC determination of warfarin and acenocoumarol in raw materials and pharmaceuticals. Journal of Pharmaceutical and Biomedical Analysis.1998;17(4-5):891-5.

8. Devries JX and Schmitz-kummer E. Direct column liquid chromatographic enatiomer separation of the coumarin anticoagulants Phenprocoumaron, Warfarin, Acenocoumarol and metabolites on an $\alpha$-1acid glycoprotein chiral stationary phase. J Chromatogr Sci. 1993;644(2):315-20.

9. Denooz R, Douamba Z and Charlier C. Fatal intoxications by acenocoumarol, phenprocoumon and warfarin: method validation in blood using the total error approach. Journal of Chromatography B Analytical Technologies in the Biomedical and Life Sciences. 2009;877(23):2344-8.

10. Roux $\mathrm{YL}$ and Richard $\mathrm{J}$ Determination of acenocoumarol in plasma and urine by double radioisotope derivative analysis. Journal of Pharmaceutical Sciences. 1977;66(7);997-1000.

11. Vecchionea G, Casettab B, Tomaiuoloa M, Grandonea E and Margaglionea M. A rapid method for the quantification of the enantiomers of Warfarin, Phenprocoumon and Acenocoumarol by two-dimensional-enantioselective liquid chromatography/electrospray tandem mass spectrometry. Journal of Chromatography B. 2007;850:507-14.

12. Guyon F, Dupeyron JP and Fabiani P. Assay of Acenocoumarol (Sintrom) in the plasma by liquid chromatography. Ann Biol Clin. 1985;43(5):743-5.

13. $\mathrm{ICH}$, Q2B Validation of Analytical Procedure; Methodology, International Conference of Harmonization for Technical requirements for the Registration of Drugs for Human use, Geneva, Switzerland, May 1997.

14. International Conference on Harmonisation (ICH) of Technical Requirements for Registration of Pharmaceuticals for Human Use: Harmonised Triplicate Guideline on Validation of Analytical Procedures: Methodology, Recommended for Adoption at Step 4 of the ICH Process on November 1996 by the ICH Steering Committee, IFPMA, Switzerland.

\section{SUMMARY}

- A Validated method was developed for estimation of nicoumalone in bulk and tablet dosage form By RP-HPLC method.

- A mixture of Acetonitrile: Potassium dihydrogen phosphate buffer $10 \mathrm{mM}$, pH 6 adjusted using $0.1 \mathrm{~N} \mathrm{KOH}$ in 50:50 v/v ratio used as mobile phase.

- The retention time of nicoumalone found to be 4-5 minutes.

- The developed method was validated as per ICH

- guidelines.

- All the validation parameters were found to be well within the acceptance criteria.

- These results show the method could find practical application as a quality control tool for analysis of the drug in its Tablet dosage forms in quality control laboratories. 